\title{
Introduction of a prognostic biomarker to strengthen risk stratification of acutely admitted patients: rationale and design of the TRIAGE III cluster randomized interventional trial
}

Andreas Sand $\varnothing^{1 *}$, Martin Schultz ${ }^{1,2}$, Jesper Eugen-Olsen², Lars Simon Rasmussen ${ }^{3}$, Lars Køber ${ }^{4}$, Erik Kjøller ${ }^{1}$, Birgitte Nybo Jensen ${ }^{5}$, Lisbet Ravn ${ }^{6}$, Theis Lange ${ }^{7}$ and Kasper Iversen ${ }^{1}$

\begin{abstract}
Background: Several biomarkers have shown to carry prognostic value beyond current triage algorithms and may aid in initial risk stratification of patients in the emergency department (ED). It has yet to be established if information provided by biomarkers can be used to prevent serious complications or deaths. Our aim is to determine whether measurement of the blood level of the biomarker soluble urokinase plasminogen activator receptor (suPAR) can enhance early risk stratification leading to reduced mortality, lower rate of complications, and improved patient flow in acutely admitted adult patients at the ED. The main hypothesis is that the availability of suPAR can reduce all-cause mortality, assessed at least 10 months after admission, by drawing attention towards patients with an unrecognized high risk, leading to improved diagnostics and treatment.
\end{abstract}

Methods: The study is designed as a cross-over cluster randomized interventional trial. SuPAR is measured within $2 \mathrm{~h}$ after admission and immediately reported to the treating physicians in the ED. All ED physicians are educated in the prognostic capabilities of suPAR prior to the inclusion period. The inclusion period began January $11^{\text {th }} 2016$ and ends June $6^{\text {th }}$ 2016. The study aims to include 10.000 patients in both the interventional and control arm. The results will be presented in 2017.

Discussion: The present article aims to describe the design and rationale of the TRIAGE III study that will investigate whether the availability of prognostic information can improve outcome in acutely admitted patients. This might have an impact on health care organization and decision-making.

Trial registration: The trial is registered at clinicaltrials.gov (ID NCT02643459, November 13, 2015) and at the Danish Data Protection agency (ID HGH-2015-042 I-Suite no. 04087).

Keywords: Risk stratification, Triage, Biomarkers, SuPAR, Emergency medicine, Acute patients

\footnotetext{
* Correspondence: andreas.hoejrup.sandoe.kristensen.01@regionh.dk

'Department of Cardiology, Herlev Hospital, University of Copenhagen,

Herlev Ringvej 75, 2730 Herlev, Denmark

Full list of author information is available at the end of the article
} 


\section{Background}

Risk stratification with triage of patients admitted to the emergency department (ED) plays a pivotal role in ensuring that the most acutely sick patients are cared for first [1]. Existing triage algorithms are all based on a combination of the patients' vital signs and primary symptoms $[1,2]$. Several retrospective studies have identified biomarkers that provide prognostic information which goes beyond the current triage utilized in the EDs [3-10].

Whether the implementation of a prognostic biomarker in initial risk stratification of acutely admitted patients translates into better management and treatment of high risk patients and actually decreases mortality, morbidity, admissions or readmissions has yet to be shown.

Soluble urokinase plasminogen activator receptor (suPAR) is a prognostic biomarker with potential use in the EDs. The suPAR blood level reflects immune activation and it is strongly associated with mortality and presence, prognosis and severity of a broad variety of acute and chronic diseases $[8,11-18]$, and it is also a predictor of disease development in the general population ([19]; Rasmussen et al.: suPAR in Acute Care: Associated with Disease Severity, Readmission, and Mortality, in review). As an unspecific biomarker with strong prognostic value across morbidities, suPAR might be a useful biomarker for risk stratification in an ED, as the staff can target intervention, resources, and clinical focus where most beneficial.

The primary aim of the study is to evaluate whether the availability of an unspecific biomarker (suPAR) as a supplement to risk stratification of unselected acutely admitted patients can reduce all-cause mortality.

\section{Design}

\section{Study design}

The TRIAGE III study is an open cross-over cluster randomized, parallel interventional two center trial on the effect of suPAR level measurements in the ED. Enrolment began January 11th 2016 and inclusion is planned to be completed June 6th 2016. The study is designed as two clusters (two EDs at two hospitals in the capital region of Denmark) around three cycles of three weeks in a 1:1 allocation ratio of intervention versus control at each hospital (Table 1). SuPAR level measurement is included in the standard blood work of all eligible patients acutely admitted in the interventional period. In the control period the suPAR level is not measured.

Table 1 Trial structure

\begin{tabular}{lllllll}
\hline Cycle & 1 & 2 & 3 & 4 & 5 & 6 \\
\hline Cluster 1 & +suPAR & Control & +suPAR & Control & +suPAR & Control \\
Cluster 2 & Control & +suPAR & Control & +suPAR & Control & +suPAR
\end{tabular}

Each cycle consists of three weeks with or without suPAR measurements in the ED $E D$ emergency department, SUPAR soluble urokinase plasminogen activator receptor

\section{Study hypothesis}

The main hypothesis is that the introduction, fast measurement and immediate reporting of the suPAR level to attending physicians in the EDs will be associated with a reduction in all-cause mortality at least 10 months after admission.

\section{Outcomes}

The primary outcome is all-cause mortality assessed on $6^{\text {th }}$ of April 2017, 10 months after inclusion of the last patient. Secondary outcomes are listed in Table 2.

\section{Eligibility criteria}

All patients presenting acutely to the ED and have blood work done including hemoglobin, $\mathrm{C}$-reactive protein and creatinine within $6 \mathrm{~h}$ of registration are included. Patients presenting in Pediatric, Gynecological or Obstetric units are excluded.

\section{Quantification of suPAR}

Blood samples (6 mL EDTA plasma tubes) for measurement of plasma suPAR are drawn along with the routine blood work. For quantification of suPAR, blood collection tubes are spun for $60 \mathrm{~s}$ at $6000 \mathrm{RPM}$. $10 \mu \mathrm{L}$ of plasma is added to a prefabricated tube containing $100 \mu \mathrm{L}$ of running buffer. Using a $60 \mu \mathrm{L}$ pipette, the plasma and buffer are mixed by pipetting the solution up and down 5 times. From this mixture, $60 \mu \mathrm{L}$ is added to the suPARnostic Quick Triage stick, a lateral flow device. After $20 \mathrm{~min}$, the lateral flow device is visually inspected for test and control line, and the suPAR test line quantified using a suPARnostic Quick test devise reader (Qiagen, Germany) [20].

According to the test manufacturer (ViroGates A/S, Birkeroed, Denmark), the limit of Detection (LOD) for the suPARnostic quick test is $0.3 \mathrm{ng} / \mathrm{mL}$. The limit of quantification (LOQ) is $2 \mathrm{ng} / \mathrm{mL}$ defined at the lowest concentration with a CV\% that does not exceed $25 \%$. The intra- and interserial measured CV\% on 5 samples $\mathrm{x} 4$ concentrations $(2.0 ; 4.0 ; 8.4 ; 13.7 \mathrm{ng} / \mathrm{mL})$ measured on the same day or with 5 days interval is less than $25 \%$. The $\mathrm{r}^{2}$ of the suPARnostic Quick Test compared to the

Table 2 Secondary outcomes

\begin{tabular}{ll}
\hline Secondary outcomes & Timeframe \\
\hline All-cause mortality after index admission & 30 days \\
Proportion of patients discharged from the ED & $24 \mathrm{~h}$ \\
Proportion of patients admitted to the ICU & 30 days \\
Incidence of new cancer diagnoses & 10 months after \\
& inclusion ends \\
Admission length & 30 days \\
\hline
\end{tabular}

A readmission is defined as any subsequent patient hospital admission within 30 days of index admission

$E D$ emergency department, $I C U$ intensive care unit 
suPARnostic ELISA is 0.875 [21]. Analysis of suPAR level is handled by trained medical students according to the manufacturer's instructions, available on-site full-time for non-stop inclusion of eligible patients. All suPAR levels are analyzed as quickly as possible and always within two hours following blood sampling and immediately reported.

\section{Information to physicians}

The suPAR level is presented to the attending physicians through the electronical systems LABKA, OPUS and Cetrea. LABKA II (v. 2.5.0.H2, Computer Sciences Corporation $(\mathrm{CSC})$ ) is the clinical laboratory information system used to request blood work and view results from laboratory analysis. OPUS (OPUS Arbejdsplads, v. 2.5.0.0, Computer Sciences Corporation (CSC) is the electronical database of medical records. The emergency wards in the EDs are monitored by the Cetrea system presented by several large screen monitors in the ED and presents a rough overview of the ward (patient data and status, possible diagnosis, route of admission) used by physicians and nurses.

Prior to the study, all physicians working in the emergency department have been informed in writing about the prognostic abilities of suPAR in unselected patients, and in regard to specific diagnoses in the form of a review of published literature, as well as pocket cards providing unadjusted mortality rates from 10.000 patients from similar EDs (see Figs. 1 and 2) ([19]; Rasmussen et al.: suPAR in Acute Care: Associated with Disease Severity, Readmission, and Mortality, in review). Furthermore, the physicians working at each specialty in the ED have attended presentations where the prognostic significance of suPAR levels and associations with morbidity and mortality were elaborated.

To assess the quality of our data, and whether the physicians received and considered the suPAR level in the initial evaluation of patients, a questionnaire is sent to 200 randomly selected physicians at the participating hospitals (Table 3 ).

\section{Data collection}

Results of blood sample analyses including suPAR level will be obtained from the LABKA II database. Using the unique Danish central person registration number (CPRnumber), data will be transferred to "Statistics Denmark" [22] and merged with data from central registries. Demographic data and mortality will be obtained from the Central Civil Registry where all residents in Denmark are registered. Data on admissions, discharges, and diagnoses will be obtained from the National Patient Registry (NPR). NPR contains information coded according to the International Statistical Classification of Disease, 10th revision (ICD-10) on primary diagnosis of discharge (Adiagnosis) and comorbidity (B-diagnoses). In the data analysis, the suPAR level from the index admission will be linked with the data above to examine the primary and secondary outcomes.

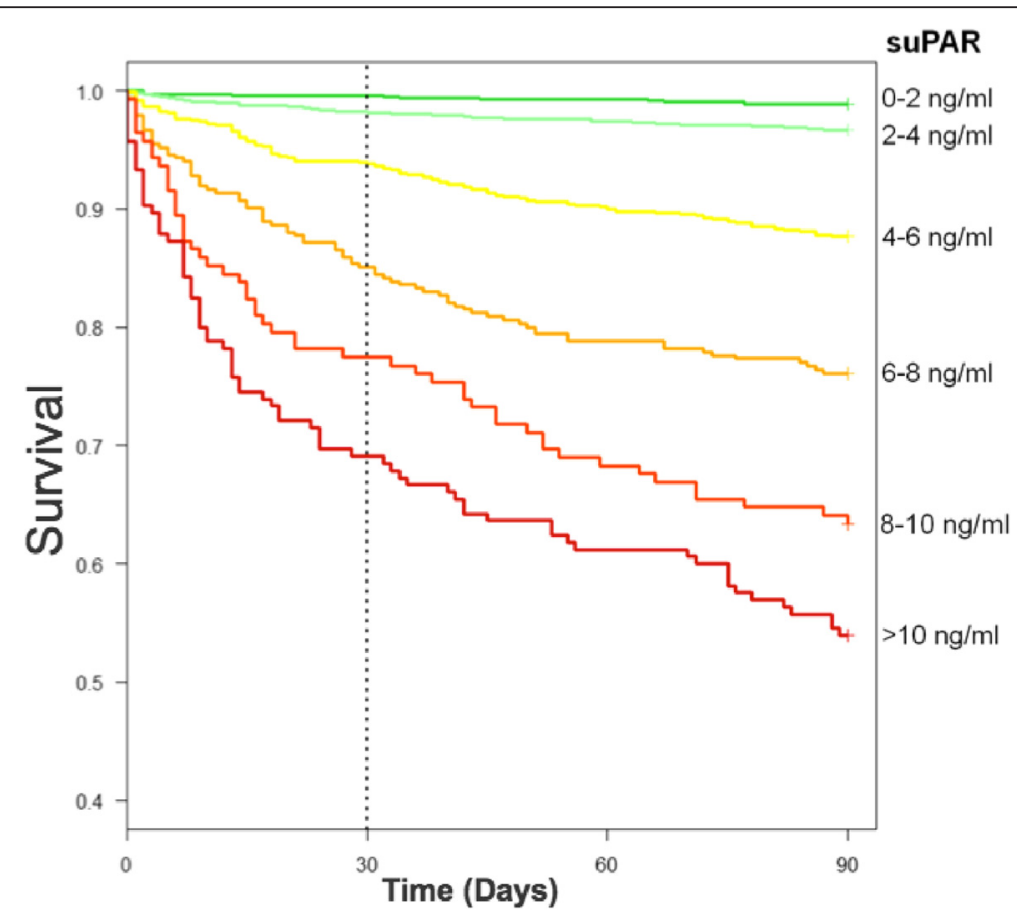

Fig. 1 Kaplan-Meier plot of survival stratified by age- and sex-specific suPAR hextiles. Data from the emergency departments at Copenhagen University Hospital Hvidovre and North Zealand Hospital $(n=9591)$ ([19]; Rasmussen et al.: suPAR in Acute Care: Associated with Disease Severity, Readmission, and Mortality, in review). SuPAR = soluble urokinase plasminogen activator receptor 

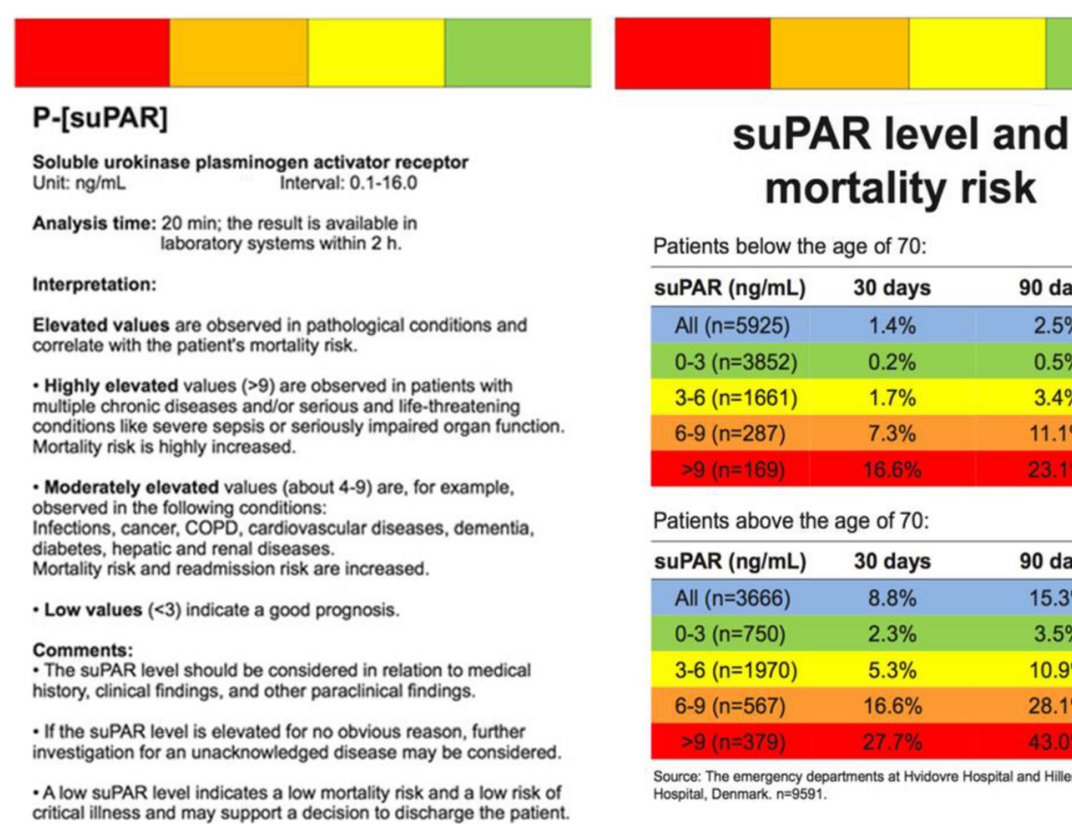

\begin{tabular}{|c|c|c|}
\hline suPAR (ng/mL) & 30 days & 90 days \\
\hline All $(n=5925)$ & $1.4 \%$ & $2.5 \%$ \\
\hline $0-3(n=3852)$ & $0.2 \%$ & $0.5 \%$ \\
\hline $3-6(n=1661)$ & $1.7 \%$ & $3.4 \%$ \\
\hline $6-9(n=287)$ & $7.3 \%$ & $11.1 \%$ \\
\hline$>9(n=169)$ & $16.6 \%$ & $23.1 \%$ \\
\hline \multicolumn{3}{|c|}{ Patients above the age of 70 : } \\
\hline suPAR $(\mathrm{ng} / \mathrm{mL})$ & 30 days & 90 days \\
\hline All $(n=3666)$ & $8.8 \%$ & $15.3 \%$ \\
\hline $0-3(n=750)$ & $2.3 \%$ & $3.5 \%$ \\
\hline $3-6(n=1970)$ & $5.3 \%$ & $10.9 \%$ \\
\hline $6-9(n=567)$ & $16.6 \%$ & $28.1 \%$ \\
\hline$>9(n=379)$ & $27.7 \%$ & $43.0 \%$ \\
\hline
\end{tabular}

Fig. 2 Pocket card given to all physicians in the ED illustrating sUPAR level interpretation and mortality risk stratified by suPAR intervals. $\mathrm{ED}=$ emergency department, suPAR = soluble urokinase plasminogen activator receptor, $\mathrm{COPD}=$ chronic obstructive pulmonary disease

\section{Power calculation}

In a previous unpublished study of patients acutely admitted to the ED at Copenhagen University Hospital North Zealand, Denmark, 12-month mortality was $12.7 \%$ and the frequency of readmissions was $16 \%$ within 5 months.

The main hypothesis is to assess if all-cause mortality at 10 months after admission is lower when the biomarker is measured on acutely admitted patients. Using a $5 \%$ level of significance and a power of $80 \%$, we will need a sample of 7340 patients in each randomization group to detect an absolute risk reduction in mortality at least 10 months after admission of $1.5 \%$.

The emergency departments at Copenhagen University Hospital Herlev and Copenhagen University Hospital Bispebjerg have a catchment area of 447.000 and 454.000 inhabitants, respectively. Based on the figures from the EDs of the hospitals included, approximately 170 patients are admitted on a daily basis. During the intervention cycles, approximately 10.710 patients will be admitted and

Table 3 Questions included in the electronical questionnaire

Did you see the suPAR level of your patient?
Did you feel informed in the prognostic ability of suPAR?

How often did you include suPAR in your combined assessment of your patient?

How often did the suPAR level influence your clinical decision?

How often were you surprised of a high suPAR level?

How often were you surprised of a low suPAR level?

SUPAR soluble urokinase plasminogen activator receptor available for suPAR measurements while 10.710 patients will be admitted in the control cycles. Thus, we anticipate to recruit $47 \%$ more patients than what is required with individual randomization, and this increase is deemed sufficient to account for intra-cluster dependency.

\section{Statistical analysis}

Patients admitted in each intervention or control cycle will be followed as a single cohort and data will be analyzed as randomized. The two groups will be assessed for comparability of the following variables: age, sex, and Charlson score. Differences in mean age of more than 5 years and/ or an absolute Charlson Comorbidity Index score of 2 or more will be adjusted for in the final analysis.

Patient data will be analyzed according to what arm of the trial the patient is admitted to during index admission according to the randomization scheme (Table 1) corresponding to the intention-to-treat principle.

A weighted Cox model will be used to compare mortality at 10 months after inclusion of the last patient. Patients are censored if their first readmission is in the opposite group of their index admission. As this censoring is likely to be dependent censoring (a readmission is rarely a positive prognostic signal), we will employ Inverse Probability of Censoring Weighting (IPCW) where patients readmitted to their own treatment group will be up-weighted to compensate. We will employ stabilized weights such that the reweighted sample has the same implied sample size throughout follow-up. Due to the design, time since index admission is the only covariate 
that needs to be included in the weights. Reweighing will be done for every two weeks of follow-up. We will not censor nor reweight for 2 nd or later readmissions, since the weights would become highly unstable and it is not likely that the presence or absence of an initial suPAR measurement will be important for clinical decisions at this stage.

Furthermore, a traditional intention-to-treat analysis will be performed. Notable difference between the results of the two analysis strategies will be considered critically.

Kaplan-Meier plots will be used to illustrate survival. Unpaired T-test will be used to compare length of stay. $P<0.05$ will be considered significant.

Subgroup analysis of the following groups will be performed: patients aged 65 year and above, and patients discharged with diagnoses of surgical conditions, cancer, infections, and cardiovascular disease.

\section{Discussion}

Rapid and safe risk stratification is necessary and an important task in emergency medicine. Identifying patients at high and low risk shortly after admission can guide clinical decision-making towards the patients in need, regarding treatment, observation and allocation of resources. Several studies have suggested biomarkers as a supplement to enhance risk stratification; however they have only been studied retrospectively $[3-7,9,10]$, why an interventional study is both warranted and required, in order to quantify the effects of implementing a prognostic biomarker in emergency medicine. The current study is to our knowledge the first of its kind, and focuses on whether the availability of a prognostic biomarker influences the treatment strategy and overall prognosis of patients admitted to the ED.

SuPAR has been evaluated as a potential biomarker in the general population by 5 large studies with more than 4500 randomly selected healthy participants which showed that elevated suPAR is associated with an increased risk of cardiovascular morbidity and mortality [13, 14, 23-25]. The TRIAGE I study along with others found suPAR independently associated with short-term mortality $[12,18,19]$, and when analyzing prospective data of the TRIAGE I study, the supplementary prognostic information of suPAR was evident (Rasmussen et al.: suPAR in Acute Care: Associated with Disease Severity, Readmission, and Mortality, in review). Receiver operational characteristic (ROC) analysis in relation to 30-day mortality showed an AUC of 0.85 (95 \% CI 0.82-0.87) when triaging with suPAR and $0.62(0.58-0.66)$ when triaging with the usual triage algorithm based on vital signs and symptoms $[19,26]$. Several biomarkers have shown to carry prognostic value, for example C-reactive protein, YKL-40, Pentraxin-3, and Copeptin [26]. SuPAR was chosen in our current interventional study based on its superiority in the TRIAGE I study, which indicated that suPAR might be a very good candidate for risk stratification in the emergency setting [19].

SuPAR as a biomarker is reflective of low-grade inflammation, a key component of disease development in e.g. cancer, cardiovascular, renal, and infectious disease and it is also strongly associated with the Charlson Comorbidity Index [11-17, 27-34]. Plasma levels of suPAR are associated with factors such as smoking, alcohol consumption, and a passive lifestyle [14]. Previous studies have shown that the urokinase system is deeply integrated in the pathogenesis of atherosclerotic remodeling and plays a role in fibrinolysis, angiogenesis and immunologic function [29, 35-37]. The suPAR level reflects immune activation and the inflammatory state of the individual. The protein UPAR is primarily expressed on immunological cells like monocytes, leukocytes, endothelial cells and is thought to reflect subclinical organ damage and endothelial dysfunction $[13,25,38]$. SuPAR meets many basic biochemical criteria of an ideal biomarkers because it is stable in plasma and is not significantly affected by the circadian cycle [39].

A biomarker reflecting the level of urgency or comorbidity burden could potentially be very useful, but the value of a biomarker with a strong negative predictive value must not be underestimated [12]. The availability of a biomarker reflecting healthiness or non-urgency (e.g. low plasma suPAR level) is particularly interesting in the setting of emergency departments where crowding is a serious concern. High bed occupancy rates are associated with an increased mortality rate, delays in initiation of time-critical care and diagnosis, increased costs and an overall poor quality of care and concerns of patient safety [40-42]. Furthermore, hospitalization is associated with a number of adverse outcomes such as falls, medication errors, infections, and delirium [43, 44]. Early discharge is associated with decreased mortality and increased patient outcome, illustrated by an American and a British study that found $26 \%$ respectively one fifth of all hospitalizations were potentially avoidable [45-47], why a more efficient selection of patients without need of admission is desirable.

\section{Strengths and limitations}

The strength of this trial is the large size of the cohort, multicenter nature and inclusion of a heterogeneous cohort under a wide variety of medical and surgical settings. Due to the unselective cohort, the trial brings generalizability whereby results will be applicable to nearly all patients admitted to EDs. A learning curve for physicians must be expected when implementing a prognostic biomarker in terms of suPAR level interpretation and intervention, why physicians might refrain from discharging patients on the basis of low suPAR levels because they do not fully trust the prognostic abilities of suPAR. 
In the planned data analysis of the primary outcome, readmitted patients remain in the group to which they are primarily allocated (control vs. suPAR intervention). This increases the risk of a type 2 error as patients primarily included in the control group might have one or more following admissions where they have suPAR measured and vice versa. A possibility would be to exclude all patients that were admitted more than once during the inclusion. If this method was chosen, we would risk excluding the sickest patients, where suPAR might have the greatest value.

\section{Conclusion and clinical implications}

The TRIAGE III trial has the potential to investigate the concept of whether the availability of prognostic information can change the patient's prognosis. This concept is central in triage and several other clinical situations, and might therefore have a central impact on health care organization and decision-making. If our hypothesis is confirmed, considerations should be given towards standardizing prognostic biomarkers as routine blood work in relation to early risk stratification in the ED.

\section{Abbreviations}

COPD, chronic obstructive pulmonary disease; ED, emergency department: sUPAR, soluble urokinase plasminogen activator receptor

\section{Acknowledgements}

The authors would like to extend our gratitude to the Departments of Clinical Biochemistry at Herlev and Bispebjerg Hospital for professional assistance and support, and to Line Jee Hartmann Rasmussen for assistance with figure and pocket card creation.

Participating clusters: Herlev Hospital, University of Copenhagen, Herlev Ringvej 75, 2730 Herlev, Denmark. Bispebjerg Hospital, University of Copenhagen, Bispebjerg Bakke 23, 2400 Copenhagen, Denmark.

\section{Funding}

The trial was supported by an unrestricted grant from ViroGates A/S (Birkeroed, Denmark). The trial is driven and initiated by the researchers and the sponsor has no influence on study design, data analysis, interpretation or publication.

\section{Availability of data and materials}

The present design article contains no data to be made available. When the database is completed, it will be located on a central server at Statistics Denmark in anonymized form. After analysis and publication of all outcomes the database will be available on request.

\section{Authors' contributions}

AS, KI, MS and JEO drafted the manuscript. LSR, LK, EK, BNJ, LR and TL contributed to the discussion, reviewed and edited the manuscript. KI had the original idea for the study. All authors read and approved the final manuscript.

\section{Competing interests}

JEO is founder of ViroGates A/S, Denmark, producing the suPARnostic ${ }^{\oplus}$ assay. Copenhagen University Hospital Hvidovre, Denmark owns the patent, which is licensed to ViroGates A/S (Birkeroed, Denmark). Remaining authors have no conflicts of interest to declare.

\section{Consent for publication}

Not applicable.

\section{Ethics approval and consent to participate}

The study was presented for the Regional Ethics committee who decided that no formal approval was needed according to Danish law (ID FSP15003590). The study data management has been approved by the Danish Data Protection agency (ID HGH-2015-042 I-Suite no. 04087).

\section{Author details}

'Department of Cardiology, Herlev Hospital, University of Copenhagen, Herlev Ringvej 75, 2730 Herlev, Denmark. ${ }^{2}$ Clinical Research Centre, Hvidovre Hospital, University of Copenhagen, Kettegård Alle 30, 2650 Hvidovre, Denmark. ${ }^{3}$ Department of Anaesthesia, Rigshospitalet, University of Copenhagen, Blegdamsvej 9, 2100 Copenhagen, Denmark. ${ }^{4}$ Department of Cardiology, Rigshospitalet, University of Copenhagen, Blegdamsvej 9, 2100 Copenhagen, Denmark. ${ }^{5}$ Department of Emergency Medicine, Bispebjerg Hospital, University of Copenhagen, Bispebjerg Bakke 23, 2400 Copenhagen, Denmark. ${ }^{6}$ Department of Emergency Medicine, Herlev Hospital, University of Copenhagen, Herlev Ringvej 75, 2730 Herlev, Denmark. ${ }^{7}$ Department of Public Health, University of Copenhagen, Øster Farimagsgade 5, 1014 Copenhagen, Denmark.

Received: 30 May 2016 Accepted: 27 July 2016

Published online: 05 August 2016

\section{References}

1. Farrohknia N, Castren M, Ehrenberg A, et al. Emergency department triage scales and their components: a systematic review of the scientific evidence. Scand J Trauma Resusc Emerg Med. 2011;19:42.

2. Iversen AK, Kristensen M, Ostervig R, Forberg JL, Schou M, Iversen K. No evidence that formalized triage is superior to informally structured triage. Ugeskr Laeger. 2016;184(4):358-61.

3. Iversen K, Gotze JP, Dalsgaard M, et al. Risk stratification in emergency patients by copeptin. BMC Med. 2014;12:80.

4. Fromm R, Meyer D, Zimmerman J, et al. A double-blind, multicentered study comparing the accuracy of diagnostic markers to predict short- and long-term clinical events and their utility in patients presenting with chest pain. Clin Cardiol. 2001;24(7):516-20.

5. Meyer B, Huelsmann M, Wexberg $P$, et al. N-terminal pro-B-type natriuretic peptide is an independent predictor of outcome in an unselected cohort of critically ill patients. Crit Care Med. 2007;35(10):2268-73.

6. Maisel A, Mueller C, Nowak RM, et al. Midregion prohormone adrenomedullin and prognosis in patients presenting with acute dyspnea: results from the BACH (Biomarkers in Acute Heart Failure) trial. J Am Coll Cardiol. 2011;58(10):1057-67.

7. Schuetz $\mathrm{P}$, Hausfater $\mathrm{P}, \mathrm{Amin} \mathrm{D}$, et al. Biomarkers from distinct biological pathways improve early risk stratification in medical emergency patients: the multinational, prospective, observational TRIAGE study. Crit Care. 2015; 19:377.

8. Seymour CW, Cooke CR, Wang Z, et al. Improving risk classification of critical illness with biomarkers: a simulation study. J Crit Care. 2013;28(5): 541-8.

9. Stahli BE, Yonekawa K, Altwegg LA, et al. Clinical criteria replenish highsensitive troponin and inflammatory markers in the stratification of patients with suspected acute coronary syndrome. PLoS One. 2014;9(6):e98626.

10. Hillis GS, Zhao N, Taggart P, Dalsey WC, Mangione A. Utility of cardiac troponin I, creatine kinase-MB(mass), myosin light chain 1, and myoglobin in the early in-hospital triage of "high risk" patients with chest pain. Heart. 1999:82(5):614-20.

11. Eapen DJ, Manocha P, Ghasemzadeh N, et al. Soluble urokinase plasminogen activator receptor level is an independent predictor of the presence and severity of coronary artery disease and of future adverse events. J Am Heart Assoc. 2014;3(5):e001118.

12. Haupt TH, Petersen J, Ellekilde G, et al. Plasma suPAR levels are associated with mortality, admission time, and Charlson Comorbidity Index in the acutely admitted medical patient: a prospective observational study. Crit Care. 2012;16(4):R130.

13. Eugen-Olsen J, Andersen O, Linneberg A, et al. Circulating soluble urokinase plasminogen activator receptor predicts cancer, cardiovascular disease, diabetes and mortality in the general population. J Intern Med. 2010;268(3):296-308.

14. Lyngbaek S, Marott $J$, Sehestedt T, et al. Cardiovascular risk prediction in the general population with use of suPAR, CRP, and Framingham Risk Score. Int J Cardiol. 2013;167(6):2904-11. 
15. Raggam RB, Wagner J, Pruller $F$, et al. Soluble urokinase plasminogen activator receptor predicts mortality in patients with systemic inflammatory response syndrome. J Intern Med. 2014;276(6):651-8.

16. Suberviola B, Castellanos-Ortega A, Ruiz Ruiz A, Lopez-Hoyos M, Santibanez M. Hospital mortality prognostication in sepsis using the new biomarkers suPAR and proADM in a single determination on ICU admission. Intensive Care Med. 2013;39(11):1945-52.

17. Donadello K, Scolletta S, Taccone FS, et al. Soluble urokinase-type plasminogen activator receptor as a prognostic biomarker in critically ill patients. J Crit Care. 2014;29(1):144-9.

18. Nayak RK, Allingstrup M, Phanareth $K$, Kofoed-Enevoldsen A. suPAR as a biomarker for risk of readmission and mortality in the acute medical setting. Dan Med J. 2015;62(10):A5146.

19. Østervig RM, Køber L, Forberg JL, Rasmussen LS, Eugen-Olsen J, Iversen K. SuPAR - A future prognostic biomarker in emergency medicine. Scand J Trauma Resusc Emerg Med. 2015;23 Suppl 1:A31.

20. Colorimetric reader for suPARnostic ${ }^{\oplus}$ Quick Tests for qualitative determination of Soluble Urokinase Plasminogen Activator Receptor in human plasma http://www.virogates.com/images/files/20140805suPARnostic-Quick-Test-Reader-Edition-11.pdf Accessed 20 Feb 2016.

21. suPARnostic ${ }^{\circledast}$ Quick Triage Performance Characteristics http://www.virogates. com/images/files/20150624_suPARnostic_QT_Performance_Characteristics.pdf Accessed 20 Feb 2016.

22. Statistics Denmark https://www.dst.dk/en Accessed 18 May 2016.

23. Botha S, Fourie CM, Schutte R, Eugen-Olsen J, Pretorius R, Schutte AE. Soluble urokinase plasminogen activator receptor as a prognostic marker of all-cause and cardiovascular mortality in a black population. Int J Cardiol. 2015;184:631-6.

24. Sorensen MH, Gerke O, Eugen-Olsen J, et al. Soluble urokinase plasminogen activator receptor is in contrast to high-sensitive C-reactive-protein associated with coronary artery calcifications in healthy middle-aged subjects. Atherosclerosis. 2014;237(1):60-6.

25. Sehestedt T, Lyngbaek S, Eugen-Olsen J, et al. Soluble urokinase plasminogen activator receptor is associated with subclinical organ damage and cardiovascular events. Atherosclerosis. 2011;216(1):237-43.

26. Plesner LL, Iversen AK, Langkjaer $\mathrm{S}$, et al. The formation and design of the TRIAGE study-baseline data on 6005 consecutive patients admitted to hospital from the emergency department. Scand J Trauma Resusc Emerg Med. 2015;23:106

27. Borne Y, Persson M, Melander O, Smith JG, Engstrom G. Increased plasma level of soluble urokinase plasminogen activator receptor is associated with incidence of heart failure but not atrial fibrillation. Eur J Heart Fail. 2014; 16(4):377-83.

28. Lyngbaek S, Andersson C, Marott J, et al. Soluble urokinase plasminogen activator receptor for risk prediction in patients admitted with acute chest pain. Clin Chem. 2013;59(11):1621-9.

29. Lyngbaek S, Marott JL, Moller DV, et al. Usefulness of soluble urokinase plasminogen activator receptor to predict repeat myocardial infarction and mortality in patients with ST-segment elevation myocardial infarction undergoing primary percutaneous intervention. Am J Cardiol. 2012;110(12):1756-63.

30. Persson $\mathrm{M}$, Ostling $\mathrm{G}$, Smith $\mathrm{G}$, et al. Soluble urokinase plasminogen activator receptor: a risk factor for carotid plaque, stroke, and coronary artery disease. Stroke. 2014;45(1):18-23.

31. Gumus A, Altintas N, Cinarka H, et al. Soluble urokinase-type plasminogen activator receptor is a novel biomarker predicting acute exacerbation in COPD. Int J Chron Obstruct Pulmon Dis. 2015;10:357-65.

32. Molkanen $T$, Ruotsalainen $E$, Thorball CW, Jarvinen A. Elevated soluble urokinase plasminogen activator receptor (suPAR) predicts mortality in Staphylococcus aureus bacteremia. Eur J Clin Microbiol Infect Dis. 2011; 30(11):1417-24.

33. Reichsoellner M, Raggam RB, Wagner J, Krause R, Hoenigl M. Clinical evaluation of multiple inflammation biomarkers for diagnosis and prognosis for patients with systemic inflammatory response syndrome. J Clin Microbiol. 2014;52(11):4063-6.

34. Zimmermann HW, Koch A, Seidler S, Trautwein C, Tacke F. Circulating soluble urokinase plasminogen activator is elevated in patients with chronic liver disease, discriminates stage and aetiology of cirrhosis and predicts prognosis. Liver Int. 2012;32(3):500-9.

35. Fuhrman B. The urokinase system in the pathogenesis of atherosclerosis. Atherosclerosis. 2012:222(1):8-14.
36. Edsfeldt A, Nitulescu M, Grufman $H$, et al. Soluble urokinase plasminogen activator receptor is associated with inflammation in the vulnerable human atherosclerotic plaque. Stroke. 2012;43(12):3305-12.

37. Blasi F, Sidenius $N$. The urokinase receptor: focused cell surface proteolysis, cell adhesion and signaling. FEBS Lett. 2010;584(9):1923-30.

38. Lyngbaek S, Sehestedt T, Marott JL, et al. CRP and suPAR are differently related to anthropometry and subclinical organ damage. Int J Cardiol. 2013; 167(3):781-5.

39. Thuno M, Macho B, Eugen-Olsen J. suPAR: the molecular crystal ball. Dis Markers. 2009;27(3):157-72.

40. Carter EJ, Pouch SM, Larson EL. The relationship between emergency department crowding and patient outcomes: a systematic review. J Nurs Scholarsh. 2014;46(2):106-15.

41. Trentino KM, Swain SG, Burrows SA, Sprivulis PC, Daly FF. Measuring the incidence of hospital-acquired complications and their effect on length of stay using CHADx. Med J Aust. 2013;199(8):543-7.

42. Madsen F, Ladelund S, Linneberg A. High levels of bed occupancy associated with increased inpatient and thirty-day hospital mortality in Denmark. Health Aff (Millwood). 2014;33(7):1236-44.

43. Collier R. Hospital-induced delirium hits hard. CMAJ. 2012;184(1):23-4.

44. Thomas EJ, Studdert DM, Burstin HR, et al. Incidence and types of adverse events and negligent care in Utah and Colorado. Med Care. 2000;38(3):261-71.

45. Basse L, Thorbol JE, LossI K, Kehlet H. Colonic surgery with accelerated rehabilitation or conventional care. Dis Colon Rectum. 2004:47(3):271-7. discussion 277-278.

46. Shewale JB, Correa AM, Baker CM, et al. Impact of a Fast-track Esophagectomy Protocol on Esophageal Cancer Patient Outcomes and Hospital Charges. Ann Surg. 2015;261(6):1114-23.

47. O'Cathain A, Knowles E, Turner J, Hirst E, Goodacre S, Nicholl J. Variation in avoidable emergency admissions: multiple case studies of emergency and urgent care systems. J Health Serv Res Policy. 2016;21(1):5-14.

\section{Submit your next manuscript to BioMed Central and we will help you at every step:}

- We accept pre-submission inquiries

- Our selector tool helps you to find the most relevant journal

- We provide round the clock customer support

- Convenient online submission

- Thorough peer review

- Inclusion in PubMed and all major indexing services

- Maximum visibility for your research

Submit your manuscript at www biomedcentral.com/submit
Ciomed Central 